\title{
Biophysical properties of cells for cancer diagnosis
}

Sharda Yadav ${ }^{1}$, Matthew J. Barton², Nam-Trung Nguyen ${ }^{1 *}$

1. Queensland Micro- and Nanotechnology Centre, Nathan Campus, Griffith University, 170 Kessels Road, Brisbane, QLD 4111, Australia.

2. Menzies Health Institute Queensland, Gold Coast Campus, Griffith University, Gold Coast, QLD 4222, Australia. 
Abstract: Biophysical properties associated with the microenvironment of a tumour has been recognised as an important modulator for cell behaviour and function. Particularly, tissue rigidity is important during 16 tumour carcinogenesis as it affects the tumour's ability to metastasis. Multiple downstream pathways are 17 affected with a difference in rigidity of the extra cellular matrix. The insight into tumour mechanosignalling represents a promising field that may lead to novel approaches for cancer diagnostics. Measurement of rigidity of the extracellular matrix or the tissue is a potential diagnostics approach for cancer detection. Altered extracellular matrix states persist for a long period of time and have lower heterogeneity compared to protein or genetic markers, therefore are more reliable as biomarkers. On the other hand, measurement of different kinase associated proteins or transcripts provide an early insight into potential transition of cells towards metastasis. Co-localization of transcriptional factors like YAP/TAZ provide an insight to determine

24 if the cells is undergoing metastatic changes. This review explains the unique biophysical properties of the tumour microenvironment that present the potential targets for the diagnosis of cancer.

Keywords: mechanotransduction, mechanical stress, cancer diagnosis, cancer cell, cancer stem cells, 


\section{Introduction}

Cancer is the second leading cause of mortality in the world and is expected to overtake cardiovascular diseases soon. Due to difficulties with early clinical and histological diagnosis, and the lack of suitable molecular markers for accurate treatments, the death rate from cancer has been increasing (Ferlay J, 2013). Only few patients die directly from the primary tumours. However, the cancer becomes very difficult to manage once it has metastasised. About $90 \%$ of metastatic cancers are terminal. Metastasis is caused by the migration of cancer cells into a new tissue. The process may occur either through nearby tissues through extracellular matrix (ECM) or far via blood or lymph to distant tissues (Fidler, 1975) like, bones, brain, liver, and lungs (Martin et al., 2000-2013). The secondary tumour in the new location is invariably different in both molecular and genetic structure in comparison to the primary tumour source. The metastatic cells are also known as circulating tumour cells (CTCs). CTCs hold information about the tumour type, its evolution and heterogeneity and hence can serve as potential diagnostic markers (Krebs et al., 2016). On the other hand, cells migrating through neighbouring tissues provide critical information on mechanobiology. During the migration process, these cells change the morphology and exert pressure to the surrounding cells. The stiffness of the cells and the tumour's microenvironment can regulate and potentiate its invasiveness (Kurniawan et al., 2016; Quail and Joyce, 2013).

Over the last few decades, cancer research has placed a significant focus on extracellular and intracellular biochemical signals in proliferation, invasion, and metastasis of cancer cells. In recent years, tumour mechanics and especially tissue rigidity has been considered as a crucial aspect of tumour progression and metastasis (Chaudhuri et al., 2018; Jaalouk and Lammerding, 2009; Wei and Yang, 2016; Yousafzai et al., 2017). To measure the stiffness of the cancer cell, Young's modulus gives the quantitative measure of elastic properties and shows the positive correlation between cell deformability and cell malignancy (Seyedpour et al., 2015; Sun et al., 2016; Xu et al., 2012). Recent study by Zhou et al had reported that the nanoindentation measures stiffness could be biomarker for cancer (Zhou et al., 2017). Tumours are often stiffer than neighbouring tissue. In soft tissues such as breast and abdomen, the stiffness of tumours is the base of clinical diagnosis and also act as a foundation for detection with imaging techniques such as MRI (Fass, 2008). The tissue of breast cancer tumour can be ten times stiffer than the normal breast tissue, and increased breast density is seen in $30 \%$ of breast tumors ( $\mathrm{Lu}$ et al., 2012). The stiffness-sensing capability of cancer and stromal cells influence cell survival and proliferation and provide new insights, so that researchers are able to develop diagnostic methods for cancer formation (Zhang et al., 2018). These diagnostic techniques have the advantage of explaining both mechanical and molecular mechanisms.

Cells sense the changes in their surrounding and transduce physical to biochemical signals, which helps to control the function, biochemical changes and gene expression (Chin et al., 2016). The physical changes in 
cell stiffness activates the integrins, which are the transmembrane proteins serving as adhesive in ECM. The activated integrins trigger a biological chain of reactions that decrease the cell contractibility (Aoudjit and Vuori, 2012; Desgrosellier and Cheresh, 2010). This process in turn provides a feedback mechanism for increasing ECM stiffness. The autocrine loop of ECM stiffness and integrin activation provides an interaction between the mechanical force on the cell and its biological processes (Huang and Ingber, 2005; Ross et al., 2013).

Cellular mechanotransduction is a process that converts mechanical signals into biochemically relevant information through a series of cellular processes (Alonso and Goldmann, 2016). The mechanical properties of the cell environment affect the cell differentiation, growth, locomotion and development (Engler et al., 2006; Rajagopalan and Saif, 2011; Yeung et al., 2005). Cells sense and exert forces on their environment, which comprise of the ECM and the basement membranes, as well as other neighbouring cells. Several generated forces are concentrated at the adhesion points between cells and the ECM, or at homotypic cell-cell adhesion. Thus, the conversion of physical stimuli into intracellular biochemical signalling or mechanotransduction should occur within the multi-protein complexes at these adhesion sites (J.H.-C.Wang and Thampatty, 2006). The possible mechanosensors include integrins, mechanosensitive ion channels, and stretch sensitive focal adhesions proteins.

The difference in the cellular mechanical properties provides an indicator of the existence and state of the disease. Changes in cancer cell from the point of initiation of a tumour to its spread to other parts of the body are observed as promising biomarker for detection of metastatic potential of these cancer cells. Also, altered ECM condition such as increased stiffness persist for a long period of time and have lower heterogeneity compared to protein or genetic markers, therefore being more reliable biomarkers. Current available technologies that can detect the cancer and metastasis based on biophysical cues are magnetic resonance elastography, ultrasound elastography, cell based sensors and atomic force microscopy (Carlsen et al., 2015; Darling et al., 2007; Pepin et al., 2015) . Although the stiffness of tissue is utilized as a basis of differentiation for tools like MRI, there are no evidences that the biochemical signals during the biophysical process being utilized for diagnostics.

This review aims to provide insights into the role of mechanical stress in cancer and the interplay between mechanical and biological processes in tumour environment as well as mechanobiology signalling. The paper also aims to provide a better insight into the biomechanical changes that occur between a healthy cell and a cancer cell. Finally, a perspective is given for the potential use of cues from biophysical processes as diagnostic markers.

\section{Cellular biology background}

\subsection{Extracellular matrix (ECM) and Integrins}


Tissues and organs are formed by cells embedded in an extracellular matrix. Cells within a tissue establish the ECM, maintain its health, alter it during adaptations and also repair it in response to disease and wound (Humphrey et al., 2014). ECM not only contributes to binding cells together, but also provides the physical scaffold for their growth, influencing their migration, differentiation, survival, homeostasis and morphogenesis (Bonnans et al., 2014). Different types of proteins and polysaccharides that are locally secreted form the matrix by organizing into a meshwork within the cell surface. The ECM consists of polysaccharide chains of repeating disaccharide units termed of glycosaminoglycans (GAGs). These are generally found to be linked to proteoglycans by covalent bonding and structural and specialized proteins such as collagen, fibrillins, elastin, fibronectin, and laminin (Mouw et al., 2014). Cells reacts to the mechanical and biochemical changes in ECM through the crosstalk between integrins and the actin cytoskeleton, to form the ECM architecture (Kim et al., 2011).

The close connection between cells and their supporting matrix is operated by the cell-matrix receptors. The cells organize the matrix and the matrix in turn influence the cell fate through the exchange of mechanical signals from the matrix to the cytoskeleton and vice versa. The interacting point between cells and their ECM is called the focal adhesion point. Cell sense their environment using specific receptors and respond to mechanical cues and induce biochemical signals from the ECM. This sensing process is a combination of mechanical and chemical systems like adhesion via integrins, focal points, molecular motors and the cytoskeletal network. Therefore, when a disease such as cancer occurs, the ECM is strongly involved (Clause and Barker, 2013; Ingber, 2008; Kumar and Weaver, 2009; Rozario and DeSimone, 2010).

Integrins are the family of adhesion proteins that regulates adhesion, progression and invasion of cancer cells. Integrins bind directly to the focal adhesion points in ECM providing the traction for motility and invasion of cancer (Figure 2). Integrin bridges two neighbouring cells and triggers intracellular signalling. Two common receptors activated by integrin are the cytokine receptors and the growth factor receptors. The communication between the growth factor receptors and the integrin leads to the hypothesis that the crosstalk between these receptors is necessary for tumour growth and invasion. They are also involved on tumour cell invasion by regulating the localisation and function of matrix- degrading proteases, for example, metalloprotease 2 and urokinase- type plasminogen activator (Ata and Antonescu, 2017; Eliceiri, 2001).

After the binding of ligands, integrins form clusters on the cell surface at focal adhesion points and serve as the origin for signal transduction from the ECM to intracellular signalling pathways. Although integrins do not possess any kinase activity, they are capable of recruiting various kinases, such as focal adhesion kinases (FAKs), integrin linked kinase (ILK) and Src family kinases (SFKs). These proteins play an active role in processes such as migration of cells, activation of Rho-GTpases, cross talk of integrins with growth factor and overturning the cellular adhesion (Srichai and Zent, 2010; Wozniak et al., 2004). The Rho GTPase contribute 
131 cell mechanics, Rho stimulate cellular contractility by activating Rho-associated kinase (ROCK) (Parri and 132 Chiarugi, 2010; Totsukawa et al., 2000).

Rho regulates the secretion of soluble matrix metalloproteinases (MMPs) like lysophosphatidic acid and transforms growth factor beta (TGF- $\beta$ ) in the tumour cells, which in turn facilitate matrix remodelling (Kumar and Weaver, 2009; Parri and Chiarugi, 2010). A typical example is the formation of cancer-associated fibroblasts (CAFs) where secreted MMPs promotes remodelling of stromal fibroblast. The isometric tension exerted on these fibroblasts enhances matrix stiffening, that triggers the formation of stress fibre and integrin mediated activation of SFK at focal points. The increase of Src signalling then promotes the nuclear accumulation of the Yes-associated protein YAP and the transcriptional co-activator TAZ, which in turn increases expression of actin modulating proteins and stabilizing actinomysoin proteins. This "continuous positive feedback loop" promotes ECM stiffening that in turn facilitates the conversion of stromal fibroblast to cancer associated fibroblasts (Calvo et al., 2013; Katsumi et al., 2004; Ross et al., 2013).

\subsection{Cell stiffness in cancer}

The spread of cells is determined by the change in the stiffness of the cell. Cellular stiffness as biomarker of relative metastatic potential has been demonstrated in ovarian cancer (Xu et al., 2012), breast cancer (Parikh et al., 2018) and prostate cancer (Reid et al., 2017). When a cell transforms from a normal mature cell to an immortal cancer cell, the cytoskeleton transforms into more irregular state from a rigid structure. The changes on the cytoskeleton are evident as malignant cells replicate more and are very motile; which is usually inhibited by a rigid cytoskeleton. Considering this fact, the changes in the cytoskeleton would generally reflect the overall mechanical properties of the cell. Thus, measuring the cellular rigidity provides information to differentiate as a normal or a cancerous cell, and is potentially a new biological marker. Differential sorting of these cells has also demonstrated the response to therapy where stiff cells are more sensitive to chemotherapy compared to softer cells (Islam et al., 2018).

Multiple techniques have been used to probe the mechanical properties of cancer cells. Most cell stretching approaches include the use of laser/ optical tweezers, mechanical microplate stretcher, microfluidic platform and micropipette aspiration (Bao and Suresh, 2003; Suresh, 2007). A microfluidic cell stretching platform was used for studying the effects of exerted stress on prostatic normal tissue-associated fibroblasts, where the authors noted that the mechanical force leads to the alignment of fibronectin secreted from stretched normal tissue-associated fibroblasts (Berrueta et al., 2018). Furthermore, Swaminathan et al. used a magnetic tweezers to demonstrate an inverse power-law relationship between cancer cell invasion and cell stiffness correlation between mechanical properties of cancer cells and their metastatic potential. As cancer cells become more invasive, they show softer mechanical characteristics which cause cell deformation and shape changes suitable for a metastatic population (Swaminathan et al., 2011). Zhang et al. have reported a novel method to measure 
the mechanical properties of cancer cells, which could be one of the criteria that help for determining whether the tissue contains lesions at the single cell level (Zhang et al., 2016). This technique could potentially be important for early prevention and accurate diagnosis of diseases.

\subsection{Hippo pathway and role of YAP/TAZ in cancer}

The Hippo signal pathway (Figure 2) has been identified for the first time in Drosophila melanogaster, controlling the size of organ through mechanisms like cell proliferation and apoptosis (Gateff, 1978; Xu et al., 1995). This signal pathway plays a vital role in the behaviour of stem cells; controlling the growth, the suppression and the regeneration of organ, and of potentially carcinogenesis (Gaspar and Tapon, 2014). The core of the mammalian hippo pathway consists of a kinase cascade, transcription coactivators, and their DNAbinding partners. The two kinases, namely mammalian Ste20-like kinases (MST) and large tumour suppressor kinase (LATS), control the activity of these two closely related transcriptional co-activators, the YAP and TAZ through phosphorylation at a specific site, which is Serine residue in the amino-terminal region of YAP-TAZ.

The transcriptional co-activators YAP/TAZ are responsible for biomechanical answer to cell shape and ECM elasticity (Totaro et al., 2018) .YAP and TAZ nuclear localization are regulated by mechanical cues that include cell-cell adhesion, and cell mechanosensing of ECM physical properties or stretching. The function of YAP/TAZ is necessary for the regulation of cell proliferation, differentiation, stem cell function and organ size. Current interest in molecular biology of YAP/TAZ has gained momentum through the realization that YAP/TAZ is highly active within human tumours(A.Warren et al., 2018). Some mouse models have indicated that the inactivation of YAP/TAZ in adult organs such as breast (Chen et al., 2014), intestine (Cai et al., 2010) and pancreas (Zhang et al., 2014) increase the emergence or progression of tumours (Azzolin et al., 2014; Harvey et al., 2013; Zanconato et al., 2016a).

\section{Cellular Mechanotranduction}

Direct interactions between cells themselves, and cells and the ECM, are critical to the development and function of multicellular organisms (Khalili and Ahmad, 2015). The key cell surface receptors involved during the attachment of cells to the ECM are integrins. Integrins are the main receptors connecting the cytoskeleton to ECM have a close relation to external force and transmit generated mechanical stresses across the plasma membrane. Apart from providing the structural stability, integrins also serve as receptors for intercellular signalling pathways. Many actions like cell adhesion, gene expression and response to triggers are mediated by integrins. (Geiger and Yamada, 2011). As mentioned earlier, the nonreceptor protein- tyrosine kinase called FAK plays an important role in integrin signalling. Along with FAK, nonreceptor protein-tyrosine kinases are also involved in signaling via integrin and focal adhesion points. (Matsui et al., 2012). They simultaneously 
act for signalling via integrin receptors (Jansen et al., 2015). As integrins also regulate the signalling pathways, they are capable of signal transduction from physical signals to chemical signals (Katsumi et al., 2004). Invasion by tumour cells is mediated by integrins and ECM attachment (Ganguly et al., 2013).

Integrins are the primary receptors connecting the cytoskeleton with the ECM, making them the bridge to transmit forces and mechanical stresses across the plasma membrane (DuFort et al., 2011). As integrins also regulate signalling pathways, they are positioned to transduce physical forces into chemical signals. Integrin mediated tumour invasion is regulated by localization and activity of matrix-degrading proteases, such as matrix metalloprotease 2 (MMP2) and urokinase-type plasminogen activator (uPA). Integrin-mediated migration generally requires FAK and SFK signalling. Mechanical stimuli can open ion channels, alter proteins binding in focal adhesion and cause changes in cell morphology. Unfolding or stretching of molecules and the opening of ion channels under mechanical forces transduce a signal to downstream-signalling pathways. Some studies demonstrate a direct link between Rho/ Rock and TRP mediated signalling that illustrate that cross talk exists between mechanical signalling via integrins and mechanosensitive ion channels.

\subsection{Mechanotransduction in cancer}

Mechanotransduction of physical signals to initiate intracellular signalling pathways are recognized in numerous cancer types through a broad range of mechanisms for example, opening of ion channels, gene modulation and protein production. Glentis et al. emphasized the central role that mechanosensing plays throughout the tumour metastasis (Glentis et al., 2017). Cancer associated fibroblasts (CAFs) are the furthermost abundant stromal cells surrounding the tumour and play a role in tumour formation, progression and metastasis (Labernadie et al., 2017). Calvo et al. showed that YAP function is critical for the establishment and maintenance of CAFs. YAP is important for many pro-tumorigenic functions of CAFs, including matrix stiffening, and invasion. (Calvo et al., 2013). Glentis et al. also demonstrated that CAFs isolated from colon cancer patients promote cancer cell invasion by interacting physically and remodelling basement membrane using mechanical forces such as pulling, stretching and softening the membrane (Glentis et al., 2017).

Cancer cells express mechanoreceptors such as integrins for increased sensitivity of ECM stiffness. Usual proteins in the adhesion complex activate the Rho GTPase for cytoskeletal reorganization. This process on the other hand leads to triggering YAP/TAZ for production of cancer stem cells. The various steps proposed for the production of these CSCs are proliferation, metastasis, survival, and additional stiffness of the ECM. Another proposed mechanism is the involvement of CAFs, which directly transmit mechanical tension to cancer cells through $\mathrm{N}$ - and E-cadherin heterophilic junctions for promoting invasion. Increased stiffness in the ECM activates YAP inside the CAFs, which then promote the stiffness of the ECM on a continuous 
autocrine feedback loop. The third proposed mechanism is the process, where mechanosensitive molecules like Polycystins 1(PC1) and 2 (PC2) activate the mTOR pathway and promote proliferation, EMT, and metastasis. In fact, PC1-CTT (carboxy-terminal tail) translocates along with TAZ to the nucleus (Gargalionis et al., 2018b; Gargalionis et al., 2015; Gargalionis et al., 2016). The above three mechanisms indicate the clear link between mechanical signals and formation of drug resistant cancer stem cells, which require further studies.

During cell differentiation, a subpopulation of cells differentiates into CSCs primarily due to oncogenic hit or initiation of oncogenesis (Figure 3). CSC are created from normal stem cells or precursor cells within the tissues after mutations take place and are usually resistant to conventional treatments (Papaccio et al., 2017). It is hypothesised that these CSCs replicate indefinitely (Hao et al., 2013), initiate tumour formation and are resistant to chemotherapy (Zanconato et al., 2016b). Recent studies have shown that the mechanical properties of ECM and cell rigidity affect normal stem cell and CSC fate (Bissell and LaBarge, 2005; Gilbert et al., 2010). Stem cells including CSCs follow FAK/ phosphatidylinositol-3 kinase (PI3K)/Akt (also known as protein kinase B) pathway for cell signalling. The stem cells use contractile forces, that result in tensile stresses in the cytoskeleton. Downstream signalling via the FAK/PI3K/Akt pathway then enhances cytoskeletal reorganization in CSCs, and affects the cell shape and focal point interactions (Kallergi et al., 2007).

The involvement of FAK/PI3K/Akt pathway can be selective to certain types of cancer. For example, chemokine receptor 1 (CXCR1), which is one of the key upstream mediators of FAK/PI3K/Akt, can be selectively targeted in vitro (Ginestier et al., 2010) by phosphorylation of the FAK. This selectivity results in activation of PI3K/Akt, which plays an vital role in mechanotransduction (J.J.Waugh and Wilson, 2008). YAP/TAZ activation leads to induction of CSC properties in a relatively wide range of human cancers. Aberrant activation of YAP/TAZ via dysregulation of the Hippo pathway results in tumorigenesis and confers cancer stem cell traits that lead to anoikis resistance, epithelial mesenchymal transition, drug resistance, and metastasis. Notably, drawbacks of current CSC markers makes it difficult to isolate CSCs from bulk heterogeneous cancer cells. Consequently, identifying the CSC-specific oncogenic signalling network of $\mathrm{YAP} / \mathrm{TAZ}$ is an attractive direction to eliminate CSC and treat cancer progression (Park et al., 2018).

\section{Conclusion and Perspectives}

Cell stiffness is an important change that occurs in metastatic cells. The changes to the ECM, integrins and overall cell mechanical process define the survivability of the cell. Studies have shown that measurement of cell stiffness is a potential biomarker for possible metastasis of cells. Thus, stiffness could be a possible early 
a metastatic process of not. Therefore, biomechanical methods to measure cell rigidity may become an 261 important diagnostic method to predict cancer formation.

The earliest use of mechanobiological property for cancer diagnostics is the method Magnetic Resonance Elastography (MRE) (Pepin et al., 2015). This technique utilizes the difference in rigidity between normal and tumour cells. Multiple cancer types including breast, brain, liver, prostate, uterus are scanned for contrast in the elasticity of the tumour or its microenvironment. In this technique, stress is applied to the tumour by mechanical waves. Images are taken during subsequent deformation process. The difference in the corresponding mechanical property is correlated back to the difference in underlying tissue architecture. Despite the innate limitation of spatial resolution and the inability of MRE to resolve small tumours, this technique is one of the biggest examples, where mechanical changes of tissue are imaged for diagnostics application.

Another potential markers for cellular stiffness are the YAP/TAZ proteins (Gargalionis et al., 2018a; Panciera et al., 2017). Cancer cells sense cell rigidity with the help of integrins. Activation of integrins triggers the HIPPO pathway initiating the nuclear internalization of YAP/TAZ proteins. Tracking this internalisation may predict the potential cancer formation in cells. Tracking the YAP/TAZ internalization would be similar to an immunohistochemistry process. Sample collected with fine needle aspiration could be probed with YAP/TAZ specific antibodies and tested for their co-localization with the nucleus. A similar approach could be followed for samples collected for histopathological examination post-surgery. Internalization of YAP/TAZ could indicate the contrast between cancerous or benign tumors.

Circulating endothelial cells (CECs) have been reported as markers of cancer (Goon et al., 2006). However, CECs are not always specific to cancer but also represent vascular damage (Uta Erdbruegger et al., 2010). CECs (Rahbari et al., 2017) may have more prognostic significance in cancer than CTCs as they are phenotypically different (Cima et al., 2016). Measurement of the difference in cell rigidity would possibly tell that if the cells will undergo metastatic transformation. Measurement of rigidity could possibility be performed using cell stretching devices providing external stress (Kamble et al., 2017). Co-localization of YAP/TAZ or estimation of MMPs (Wirtz et al., 2011) levels are also potential options for differentiating normal cells with cancer cells. Proteomic or transcriptomic estimation of Rho, MMPs or Rac1 provide information on epithelial-tomesenchymal transition (EMT) of cells. Rho is upregulated in epithelial cells whereas MMPs and Rac1 are upregulated in mesenchymal cells (Friedl and Alexander, 2011).

Recently, polycystins have also been reported as mechano-transducer molecules in cancer. Their role is largely undefined. However, their co-internalization with TAZ has been reported (Gargalionis et al., 2016). 
Further investigation with these molecules may determine if they could serve as an early marker for cancer diagnostics.

Understanding the overall mechanosensing properties of cancer cells can also leads to better drug discovery or regulate patient's response to anti-cancer treatment. Involvement of YAP/TAZ in various aspects of cancer mechanobiology makes them exciting targets for cancer drug development. Moreover, the involvement of the Hippo pathway in the proliferative potential, drug resistance and metastasis of cancer cells should also be explored. The main component of the Hippo pathway YAP has recently been shown to function as both a protooncogene and a tumour suppressor (Broders-Bondon et al., 2018; Moroishi et al., 2015). There is potentially a correlation between mechanotransduction pathway and CSCs. Mechanical stimulation influences cancer growth and the biomechanical pathway (FAK/PI3K/Akt) pathway influences CSCs activity, potentially elucidating newer pathways for the treatment of drug resistance cancer (Ginestier et al., 2010; Vara et al., 2004).

In conclusion, understanding cell stiffness and mechanosensing molecules in cells and ECM are essential for discovering possible biomarkers of cancer. The added benefit of these markers is their predictive role as compared to diagnostic or prognostic markers currently employed clinically. We have previously described the different methods to induce strain to cells (Kamble et al., 2016a; Kamble et al., 2016b; Kamble et al., 2017). However, further research to measure the rigidity of cells in relation to cancer would lead new predictive biomarkers for cancer. In addition to diagnostic applications, measurement of cell rigidity and mechanobiological parameters may indicate their likelihood for differentiation into cancer. Measurement of these parameters may also predict if the cancer cells will become drug resistant. In this respect, our cell stretching technology may also be used as a tool for drug development.

\section{Acknowledgements}

This work was supported by Australian Research Council (DP170100277, DP180100055) to N.-T. N and higher degree research scholarships GUIPRS and GUPRS Scholarships to S.Y from the Griffith University.

\section{Conflict of Interest}

The authors declare no conflict of interest.

\section{References}

A.Warren, J.S., Xiao, Y., Lamar, J.M., 2018. YAP/TAZ Activation as a Target for Treating Metastatic Cancer. Cancer 10.

Alonso, J.L., Goldmann, W.H., 2016. Cellular mechanotransduction. AIMS Biophysics 3, 50-62.

Aoudjit, F., Vuori, K., 2012. Integrin signaling in cancer cell survival and chemoresistance. Chemotherapy research and practice 2012, 1-16. 
Ata, R., Antonescu, C.N., 2017. Integrins and Cell Metabolism: An Intimate Relationship Impacting Cancer. Int. J. Mol. Sci. 18, 1-31.

Azzolin, L., Panciera, T., Soligo, S., Enzo, E., Bicciato, S., Dupont, S., Bresolin, S., Frasson, C., Basso, G., Guzzardo, V., Fassina, A., Cordenonsi, M., Piccolo, S., 2014. YAP/TAZ incorporation in the beta-catenin destruction complex orchestrates the Wnt response. Cell 158, 157-170.

Bao, G., Suresh, S., 2003. Cell and molecular mechanics of biological materials. Nat. Mater. 2, 715-725.

Berrueta, L., Bergholz, J., Munoz, D., Muskaj, I., Badger, G.J., Shukla, A., Kim, H.J., Zhao, J.J., Langevin, H.M., 2018. Stretching Reduces Tumor Growth in a Mouse Breast Cancer Model. Sci Rep 8, 7864.

Bissell, M.J., LaBarge, M.A., 2005. Context, tissue plasticity, and cancer: are tumor stem cells also regulated by the microenvironment? Cancer Cell. 7, 17-23.

Bonnans, C., Chou, J., Werb, Z., 2014. Remodelling the extracellular matrix in development and disease. Nat Rev Mol Cell Biol. 15, 786-801.

Broders-Bondon, F., Ho-Bouldoires, T.H.N., Fernandez-Sanchez, M.-E., Farge, E., 2018. Mechanotransduction in tumor progression: The dark side of the force. J. Cell Biol. 217, 1571-1587.

Cai, J., Zhang, N., Zheng, Y., Wilde, R.F.d., Maitra, A., Pan, D., 2010. The Hippo signaling pathway restricts the oncogenic potential of an intestinal regeneration program. Genes Dev. 24, 2383-2388.

Calvo, F., Ege, N., Grande-Garcia, A., Hooper, S., Jenkins, R.P., Chaudhry, S.I., Harrington, K., Williamson, P., Moeendarbary, E., Charras, G., Sahai, E., 2013. Mechanotransduction and YAP-dependent matrix remodelling is required for the generation and maintenance of cancer-associated fibroblasts. Nat Cell Biol. 15, 637-646. Carlsen, J., Ewertsen, C., Sletting, S., Vejborg, I., Schafer, F.K., Cosgrove, D., Bachmann Nielsen, M., 2015. Ultrasound Elastography in Breast Cancer Diagnosis. Ultraschall in der Medizin 36, 550-562; quiz 563-555. Chaudhuri, P.K., Low, B.C., Lim, C.T., 2018. Mechanobiology of Tumor Growth. Chem Rev 118, 6499-6515. Chen, Q., Zhang, N., Gray, R.S., Li, H., Ewald, A.J., Zahnow, C.A., Pan, D., 2014. A temporal requirement for Hippo signaling in mammary gland differentiation, growth, and tumorigenesis. Genes Dev 28, 432-437. Chin, L., Xia, Y., Discher, D.E., Janmey, P.A., 2016. Mechanotransduction in cancer. Curr. Opin. Chem. Eng. 11, 77-84.

Cima, I., Kong, S.L., Sengupta, D., Tan, I.B., Phyo, W.M., Lee, D., Hu, M., Iliescu, C., Alexander, I., Goh, W.L., Rahmani, M., Suhaimi, N.-A.M., Vo, J.H., Tai, J.A., Tan, J.H., Chua, C., Ten, R., Lim, W.J., Chew, M.H., Hauser, C.A.E., Dam, R.M.v., Lim, W.-Y., Prabhakar, S., Lim, B., Koh, P.K., Robson, P., Ying, J.Y., Hillmer, A.M., Tan, M.$H ., 2016$. Tumor-derived circulating endothelial cell clusters in

colorectal cancer. Sci Transl Med. 8, 345ra389.

Clause, K.C., Barker, T.H., 2013. Extracellular matrix signaling in morphogenesis and repair. Curr. Opin. Biotechnol. 24, 830-833.

Darling, E.M., Zauscher, S., Block, J.A., Guilak, F., 2007. A thin-layer model for viscoelastic, stress-relaxation testing of cells using atomic force microscopy: do cell properties reflect metastatic potential? Biophysical journal 92, 1784-1791.

Desgrosellier, J.S., Cheresh, D.A., 2010. Integrins in cancer: biological implications and therapeutic opportunities. Nat Rev Cancer. 10, 9-22.

DuFort, C.C., Paszek, M.J., Weaver, V.M., 2011. Balancing forces: architectural control of mechanotransduction. Nature reviews. Molecular cell biology 12, 308-319.

Eliceiri, B.P., 2001. Integrin and Growth Factor Receptor Crosstalk. Circ.Res. 89, 1104-1110.

Engler, A.J., Sen, S., Sweeney, H.L., Discher, D.E., 2006. Matrix elasticity directs stem cell lineage specification. Cell 126, 677-689.

Fass, L., 2008. Imaging and cancer: a review. Mol. Oncol. 2, 115-152.

Ferlay J, S.I., Ervik M, Dikshit R, Eser S, Mathers C., 2013. GLOBOCAN 2012 v1.0, Cancer Incidence and Mortality Worldwide: IARC CancerBase No. 11. International Agency for Research on Cancer, Lyon, France.

Fidler, I.J., 1975. Mechanisms of Cancer Invasion and Metastasis, in: Becker, F. (Ed.), Biology of Tumors: Surfaces, Immunology, and Comparative Pathology. Springer US, pp. 101-131.

Friedl, P., Alexander, S., 2011. Cancer invasion and the microenvironment: plasticity and reciprocity. Cell 147, 992-1009.

Ganguly, K.K., Pal, S., Moulik, S., Chatterjee, A., 2013. Integrins and metastasis. Cell adhesion \& migration 7, 251-261. 
Gargalionis, A.N., Basdra, E.K., Papavassiliou, A.G., 2018a. Cancer mechanobiology: Effects and therapeutic perspectives. Int. J. Cancer 142, 1298-1299. Gargalionis, A.N., Basdra, E.K., Papavassiliou, A.G., 2018b. Tumor mechanosensing and its therapeutic potential. J. Cell. Biochem., 1-5.

Gargalionis, A.N., Korkolopoulou, P., Farmaki, E., Piperi, C., Dalagiorgou, G., Adamopoulos, C., Levidou, G., Saetta, A., Fragkou, P., Tsioli, P., Kiaris, H., Zizi-Serbetzoglou, A., Karavokyros, I., Papavassiliou, K.A., Tsavaris, N., Patsouris, E., Basdra, E.K., Papavassiliou, A.G., 2015. Polycystin-1 and polycystin-2 are involved in the acquisition of aggressive phenotypes in colorectal cancer. Int. J. Cancer 136, 1515-1527.

Gargalionis, A.N., Papavassiliou, K.A., Basdra, E.K., Papavassiliou, A.G., 2016. Polycystins: Mechanosensors with Diagnostic and Prognostic Potential in Cancer. Trends Mol. Med 22, 7-9.

Gaspar, P., Tapon, N., 2014. Sensing the local environment: actin architecture and Hippo signalling. Curr. Opin. Cell Biol. 31, 74-83.

Gateff, E., 1978. Malignant Neoplasms of Genetic Origin in Drosophila melanogaster. Science 200, 1448-1459. Geiger, B., Yamada, K.M., 2011. Molecular architecture and function of matrix adhesions. Cold Spring Harb Perspect Biol. 3, a005033.

Gilbert, P.M., Havenstrite, K.L., Magnusson, K.E.G., Sacco, A., Leonardi, N.A., Kraft, P., Nguyen, N.K., Thrun, S., Lutolf, M.P., Blau, H.M., 2010. Substrate Elasticity Regulates Skeletal Muscle Stem Cell Self-Renewal in Culture. Science 329, 1078- 1081.

Ginestier, C., Liu, S., Diebel, M.E., Korkaya, H., Luo, M., Brown, M., Wicinski, J., Cabaud, O., Charafe-Jauffret, E., Birnbaum, D., Guan, J.-L., Dontu, G., Wicha, M.S., 2010. CXCR1 blockade selectively targets human breast cancer stem cells in vitro and in xenografts. J. Clin. Investig. 120, 485-497.

Glentis, A., Oertle, P., Mariani, P., Chikina, A., Marjou, F.E., Attieh, Y., Zaccarini, F., Lae, M., Loew, D., Dingli, F., Sirven, P., Schoumacher, M., Gurchenkov, B.G., Plodinec, M., Vignjevic, D.M., 2017. Cancer-associated Fibroblasts Induce Metalloprotease-independent Cancer Cell Invasion of the Basement membrane. Nat. Commun. 8, 1-13.

Goon, P.K., Lip, G.Y., Boos, C.J., Stonelake, P.S., Blann, A.D., 2006. Circulating endothelial cells, endothelial progenitor cells, and endothelial microparticles in cancer. Neoplasia 8, 79-88.

Hao, J., Zhang, Y., Ye, R., Zheng, Y., Zhao, Z., Li, J., 2013. Mechanotransduction in cancer stem cells. Cell Biol. Int. 37, 888-891.

Harvey, K.F., Zhang, X., Thomas, D.M., 2013. The Hippo pathway and human cancer. Nat. Rev. Cancer 13, 246-257.

Huang, S., Ingber, D.E., 2005. Cell tension, matrix mechanics, and cancer development. Cancer Cell 8, 175176.

Humphrey, J.D., Dufresne, E.R., Schwartz, M.A., 2014. Mechanotransduction and extracellular matrix homeostasis. Nat Rev Mol Cell Biol. 15, 802-812.

Ingber, D.E., 2008. Can cancer be reversed by engineering the tumor microenvironment? Semin. Cancer Biol. 18, 356-364.

Islam, M., Mezencev, R., McFarland, B., Brink, H., Campbell, B., Tasadduq, B., Waller, E.K., Lam, W., Alexeev, A., Sulchek, T., 2018. Microfluidic cell sorting by stiffness to examine heterogenic responses of cancer cells to chemotherapy. Cell Death Dis 9, 239.

J.H.-C.Wang, Thampatty, B.P., 2006. An introductory review of cell mechanobiology. Biomechan Model Mechanobiol 5, 1-16.

J.J.Waugh, D., Wilson, C., 2008. The interleukin-8 pathway in cancer. Clin. Cancer Res. 14, 6735-6741.

Jaalouk, D.E., Lammerding, J., 2009. Mechanotransduction gone awry. Nat Rev Mol Cell Biol. 10, 63-73.

Jansen, K.A., Donato, D.M., Balcioglu, H.E., Schmidt, T., Erik H.J. Danen, Koenderink, G.H., 2015. A guide to mechanobiology: Where biology and physics meet. Biochim. Biophys. Acta 1853, 3043-3052.

Kallergi, G., Agelaki, S., Markomanolaki, H., Georgoulias, V., Stournaras, C., 2007. Activation of FAK/PI3K/Rac1 Signaling Controls Actin Reorganization and Inhibits Cell Motility in Human Cancer Cells. Cell. Physiol. Biochem. 20, 977-986.

Kamble, H., Barton, M.J., Jun, M., Park, S., Nguyen, N.-T., 2016a. Cell stretching devices as research tools: engineering and biological considerations. Lab on a chip 16, 3193-3203.

Kamble, H., Jun, M., Park, S., Barton, M.J., Vadivelu, R.K., John, J.S., Nguyen, N.-T., 2016b. An electromagnetic cell-stretching device for mechanotransduction studies of olfactory ensheathing cells. Biomed. Microdevices 18, 1-10. 
Kamble, H., Vadivelu, R., Barton, M., Boriachek, K., Ahmed, M., Park, S., Shiddiky, M.J.A., Nguyen, N.-T., 2017. An Electromagnetically Actuated Double-Sided Cell-Stretching Device for Mechanobiology Research. Micromachines 8, 256.

Katsumi, A., Orr, A.W., Tzima, E., Schwartz, M.A., 2004. Integrins in Mechanotransduction. J. Biol. Chem. 279, 12001-12004.

Khalili, A.A., Ahmad, M.R., 2015. A Review of Cell Adhesion Studies for Biomedical and Biological Applications. Int. J. Mol. Sci. 16, 18149-18184.

Kim, S.-H., Turnbull, J., Guimond, S., 2011. Extracellular matrix and cell signalling: the dynamic cooperation of integrin, proteoglycan and growth factor receptor. J. Endocrinol. 209, 139-151.

Krebs, M.G., Hou, J.-M., Ward, T.H., Blackhall, F.H., Dive, C., 2016. Circulating tumour cells: their utility in cancer management and predicting outcomes. Ther. Adv. Med. Oncol. 2, 351-365. Kumar, S., Weaver, V.M., 2009. Mechanics, malignancy, and metastasis: the force journey of a tumor cell. Cancer Metastasis Rev. 28, 113-127.

Kurniawan, N.A., Chaudhuri, P.K., Lim, C.T., 2016. Mechanobiology of cell migration in the context of dynamic two-way cell-matrix interactions. Journal of biomechanics 49, 1355-1368.

Labernadie, A., Kato, T., Brugués, A., Serra-Picamal, X., Derzsi, S., Arwert, E., Weston, A., González-Tarragó, V., Elosegui-Artola, A., Albertazzi, L., Alcaraz, J., Roca-Cusachs, P., Sahai, E., Trepa, X., 2017. A mechanically active heterotypic $\mathrm{E}$-cadherin/ $\mathrm{N}$-cadherin adhesion enables fibroblasts to drive cancer cell invasion. Nat Cell Biol. 19, 224-237.

Lu, P., Weaver, V.M., Werb, Z., 2012. The extracellular matrix: a dynamic niche in cancer progression. The Journal of cell biology 196, 395-406.

Martin, T.A., Ye, L., Sanders, A.J., Lane, J., Jiang, W.G., 2000-2013. Cancer Invasion andMetastasis: Molecular and Cellular perspective, Madame Curie Bioscience Database. Landes Bioscience, Austin (TX).

Matsui, H., Harada, I., Sawada, Y., 2012. Src, p130Cas, and Mechanotransduction in Cancer Cells. Genes \& cancer 3, 394-401.

Moroishi, T., Hansen, C.G., Guan, K.-L., 2015. The emerging roles of YAP and TAZ in cancer. Nat Rev Cancer. 15, 73-79.

Mouw, J.K., Ou, G., Weaver, V.M., 2014. Extracellular matrix assembly: a multiscale deconstruction. Nat Rev Mol Cell Biol. 15, 771-785.

Panciera, T., Azzolin, L., Cordenonsi, M., Piccolo, S., 2017. Mechanobiology of YAP and TAZ in physiology and disease. Nature reviews. Molecular cell biology 18, 758-770.

Papaccio, F., Paino, F., Regad, T., Papaccio, G., Desiderio, V., Tirino, V., 2017. Concise Review: Cancer Cells, Cancer Stem Cells, and Mesenchymal Stem Cells: Influence in Cancer Development. Stem Cells Transl Med. 6, 2115-2125.

Parikh, D., Stack, M., Wang, H., 2018. Matrix Stiffness Regulates the Fate of Breast Cancer Cells. Biophysical journal 114. Park, J.H., Shin, J.E., Park, H.W., 2018. The Role of Hippo Pathway in Cancer Stem Cell Biology. Mol Cells 41, 83-92.

Parri, M., Chiarugi, P., 2010. Rac and Rho GTPases in cancer cell motility control. Cell Commun. Signal. 8, 114.

Pepin, K.M., Ehman, R.L., McGee, K.P., 2015. Magnetic resonance elastography (MRE) in cancer: Technique, analysis, and applications. Prog Nucl Magn Reson Spectrosc 90-91, 32-48.

Quail, D., Joyce, J., 2013. Microenvironmental regulation of tumor progression and metastasis. Nat Med. 19, 1423-1437.

Rahbari, N.N., Schölch, S., Bork, U., Kahlert, C., Schneider, M., Rahbari, M., Büchler, M.W., Weitz, J., Reissfelder, C., 2017. Prognostic value of circulating endothelial cells in metastatic

colorectal cancer. Oncotarget 8, 37491-37501.

Rajagopalan, J., Saif, M.T.A., 2011. MEMS Sensors and Microsystems for Cell Mechanobiology. J Micromech Microeng. 21, 54002-54012.

480 Reid, S.E., Kay, E.J., Neilson, L.J., Henze, A.T., Serneels, J., McGhee, E.J., Dhayade, S., Nixon, C., Mackey, J.B., 481 Santi, A., Swaminathan, K., Athineos, D., Papalazarou, V., Patella, F., Roman-Fernandez, A., ElMaghloob, Y., 482 Hernandez-Fernaud, J.R., Adams, R.H., Ismail, S., Bryant, D.M., Salmeron-Sanchez, M., Machesky, L.M., Carlin, 
L.M., Blyth, K., Mazzone, M., Zanivan, S., 2017. Tumor matrix stiffness promotes metastatic cancer cell interaction with the endothelium. EMBO J 36, 2373-2389.

Ross, T.D., Coon, B.G., Yun, S., Baeyens, N., Tanaka, K., Ouyang, M., Schwartz, M.A., 2013. Integrins in mechanotransduction. Curr. Opin. Cell Biol. 25, 613-618.

Rozario, T., DeSimone, D.W., 2010. The extracellular matrix in development and morphogenesis: a dynamic view. Dev Biol. 341, 126-140.

Seyedpour, S.M., Pachenari, M., Janmaleki, M., Alizadeh, M., Hosseinkhani, H., 2015. Effects of an antimitotic drug on mechanical behaviours of the cytoskeleton in distinct grades of colon cancer cells. Journal of biomechanics 48, 1172-1178.

Srichai, M.B., Zent, R., 2010. Integrin Structure and Function, in: Zent, R., Pozzi, A. (Eds.), Cell-Extracellular Matrix Interactions in Cancer. Springer-Verlag, New York, pp. 19-41.

Sun, J., Luo, Q., Liu, L., Zhang, B., Shi, Y., Ju, Y., Song, G., 2016. Biomechanical profile of cancer stem-like cells derived from MHCC97H cell lines. Journal of biomechanics 49, 45-52.

Suresh, S., 2007. Biomechanics and biophysics of cancer cells. Acta Mater. 55, 3989-4014.

Swaminathan, V., Mythreye, K., O'Brien, E.T., Berchuck, A., Blobe, G.C., Superfine, R., 2011. Mechanical stiffness grades metastatic potential in patient tumor cells and in cancer cell lines. Cancer Res 71, 5075-5080. Totaro, A., Castellan, M., Biagio, D.D., Piccolo, S., 2018. Crosstalk between YAP/TAZ and Notch Signaling. Trends Cell Biol.

Totsukawa, G., Yamakita, Y., Yamashiro, S., Hartshorne, D.J., Sasaki, Y., Matsumura, F., 2000. Distinct roles of ROCK (Rho-kinase) and MLCK in spatial regulation of MLC phosphorylation for assembly of stress fibers and focal adhesions in 3T3 fibroblasts. J Cell Biol. 150, 797-806.

Uta Erdbruegger, Ajay Dhaygude, Marion Haubitz, Woywodt, A., 2010. Circulating Endothelial Cells: Markers and Mediators of Vascular Damage Curr Stem Cell Res Ther. 5, 294-302.

Vara, J.A.F., casado, E., Castro, J.d., Cejas, P., Belda-Iniesta, C., Gonzalez-Baron, M., 2004. PI3K/Akt signalling pathway and cancer. Cancer Treat. Rev. 30, 193-204.

Wei, S.C., Yang, J., 2016. Forcing through Tumor Metastasis: The Interplay between Tissue Rigidity and Epithelial-Mesenchymal Transition. Trends Cell Biol 26, 111-120.

Wirtz, D., Konstantopoulos, K., Searson, P.C., 2011. The physics of cancer: the role of physical interactions and mechanical forces in metastasis. Nature reviews. Cancer 11, 512-522.

Wozniak, M.A., Modzelewska, K., Kwong, L., Keely, P.J., 2004. Focal adhesion regulation of cell behavior. Biochimica et biophysica acta 1692, 103-119.

Xu, T., Wang, W., Zhang, S., Stewart, R.A., Yu, W., 1995. Identifying tumor suppressors in genetic mosaics: the Drosophila lats gene encodes a putative protein kinase. Development 121, 1053-1063.

Xu, W., Mezencev, R., Kim, B., Wang, L., McDonald, J., Sulchek, T., 2012. Cell Stiffness Is a Biomarker of the Metastatic Potential of Ovarian Cancer Cells. PloS one 7.

Yeung, T., Georges, P.C., Flanagan, L.A., Marg, B., Ortiz, M., Funaki, M., Zahir, N., Ming, W., Weaver, V., Janmey, P.A., 2005. Effects of substrate stiffness on cell morphology, cytoskeletal structure, and adhesion. Cell Motil. Cytoskeleton 60, 24-34.

Yousafzai, M.S., Coceano, G., Bonin, S., Niemela, J., Scoles, G., Cojoc, D., 2017. Investigating the effect of cell substrate on cancer cell stiffness by optical tweezers. Journal of biomechanics 60, 266-269.

Zanconato, F., Battilana, G., Cordenonsi, M., Piccolo, S., 2016a. YAP/TAZ as therapeutic targets in cancer. Curr. Opin. Pharmacol. 29, 26-33.

Zanconato, F., Cordenonsi, M., Piccolo, S., 2016b. YAP/TAZ at the Roots of Cancer. Cancer Cell 29, 783-803.

Zhang, S.X., Liu, L., Zhao, W., 2018. Targeting Biophysical Cues: a Niche Approach to Study, Diagnose, and Treat Cancer. Trends in cancer 4, 268-271.

Zhang, T., Zhao, Y., Tong, Z., Guan, Y., 2016. A novel method to calculate the mechanical properties of cancer cells based on atomic force microscopy. Acta of Bioengineering and Biomechanics 18, 19-24.

Zhang, W., Nandakumar, N., Shi, Y., Manzano, M., Smith, A., Graham, G., Gupta, S., Vietsch, E.E., Laughlin, S.Z., Wadhwa, M., Chetram, M., Joshi, M., Wang, F., Kallakury, B., Toretsky, J., Wellstein, A., Yi, C., 2014. Downstream of mutant KRAS, the transcription regulator YAP is essential for neoplastic progression to pancreatic ductal adenocarcinoma. Sci Signal. 7, ra42.

Zhou, Z.L., Sun, X.X., Ma, J., Tong, M.H., To, S.K.Y., Wong, A.S.T., Ngan, A.H.W., 2017. Actin cytoskeleton stiffness grades metastatic potential of ovarian carcinoma Hey A8 cells via nanoindentation mapping. Journal of biomechanics 60, 219-226. 


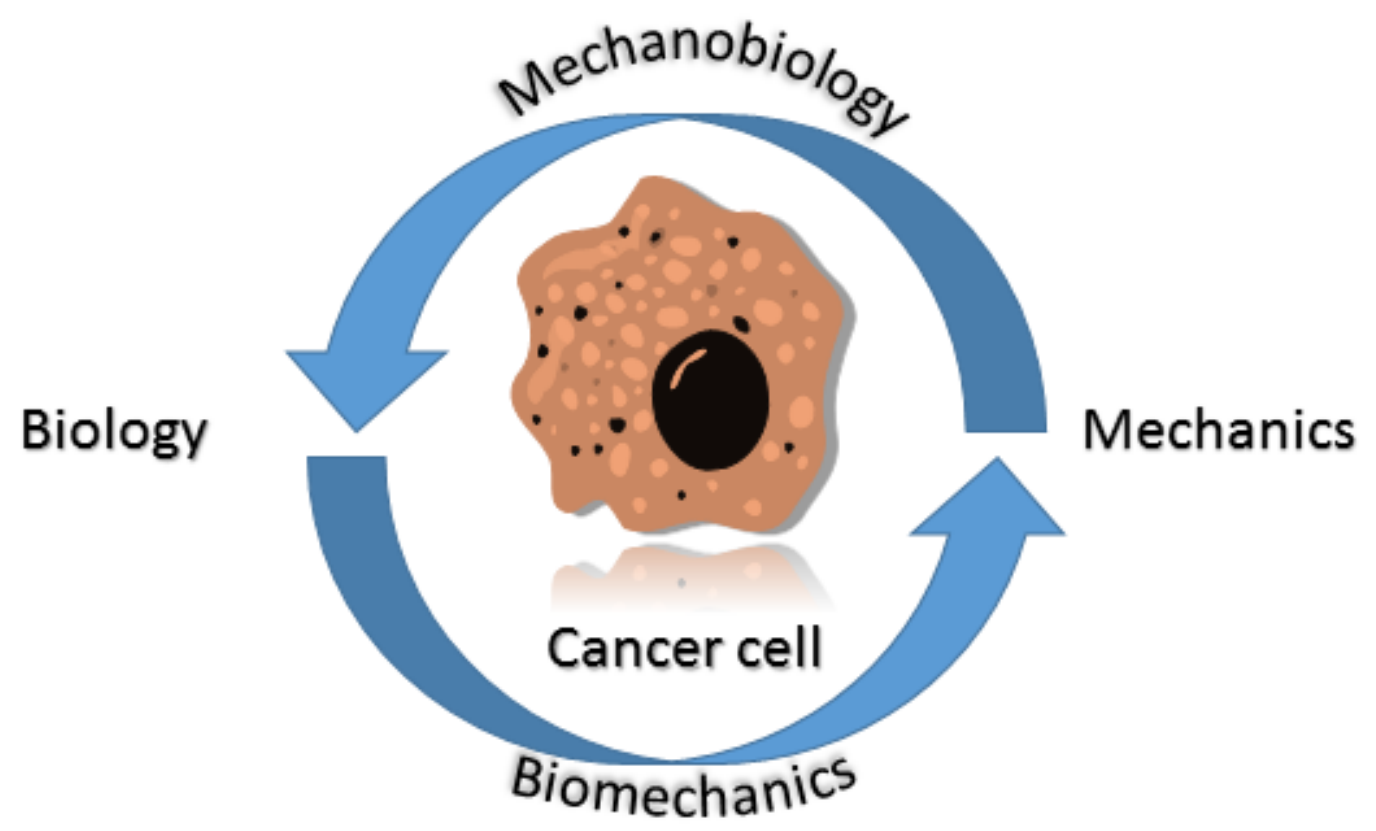

537

538 Fig 1: A graphical representation of the overall concept of the review article.

539

540

541

542

543

544

545 


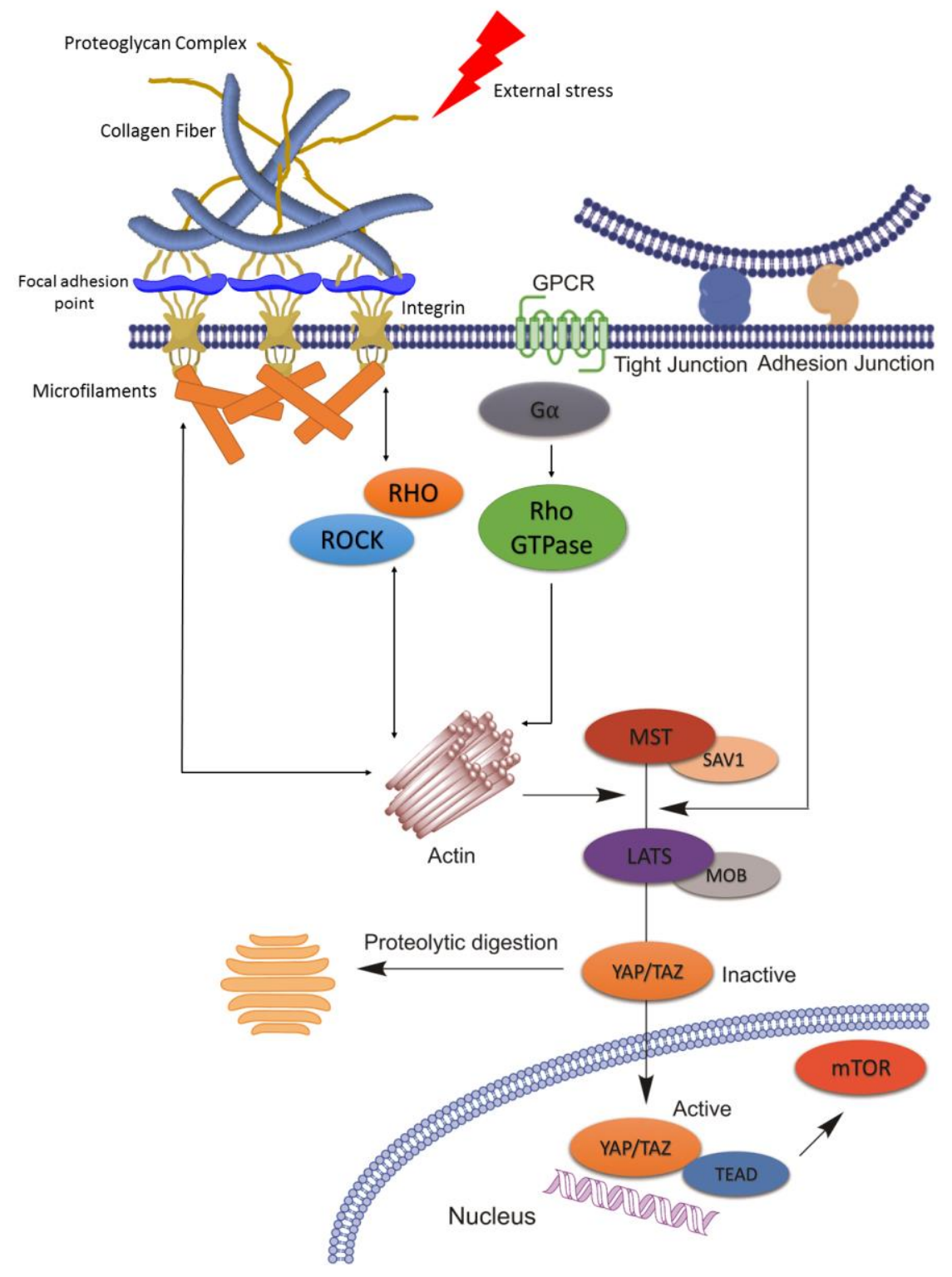

548 Figure 2. The Extra Cellular Matrix is composed of proteoglycan complex that is interwoven with 549 collagen fibers. This mesh is connected to fibronectins, which is then connected to the integrins. Integrins 550 bind directly to the focal adhesion points in ECM. Integrins help in signal transduction from the ECM to 551 intracellular signalling pathways. Hippo-YAP signal pathway is regulated by extracellular forces. 552 Extracellular stress activates the Rho GTPases, which inhibit Lats1/2 kinase activity. Activated YAP/TAZ 553 migrate inside nucleus whereas the inactive YAP/TAZ goes to proteolytic digestion. GPCRs, cellular 554 junction and cell polarity modulate the Hippo pathway. 


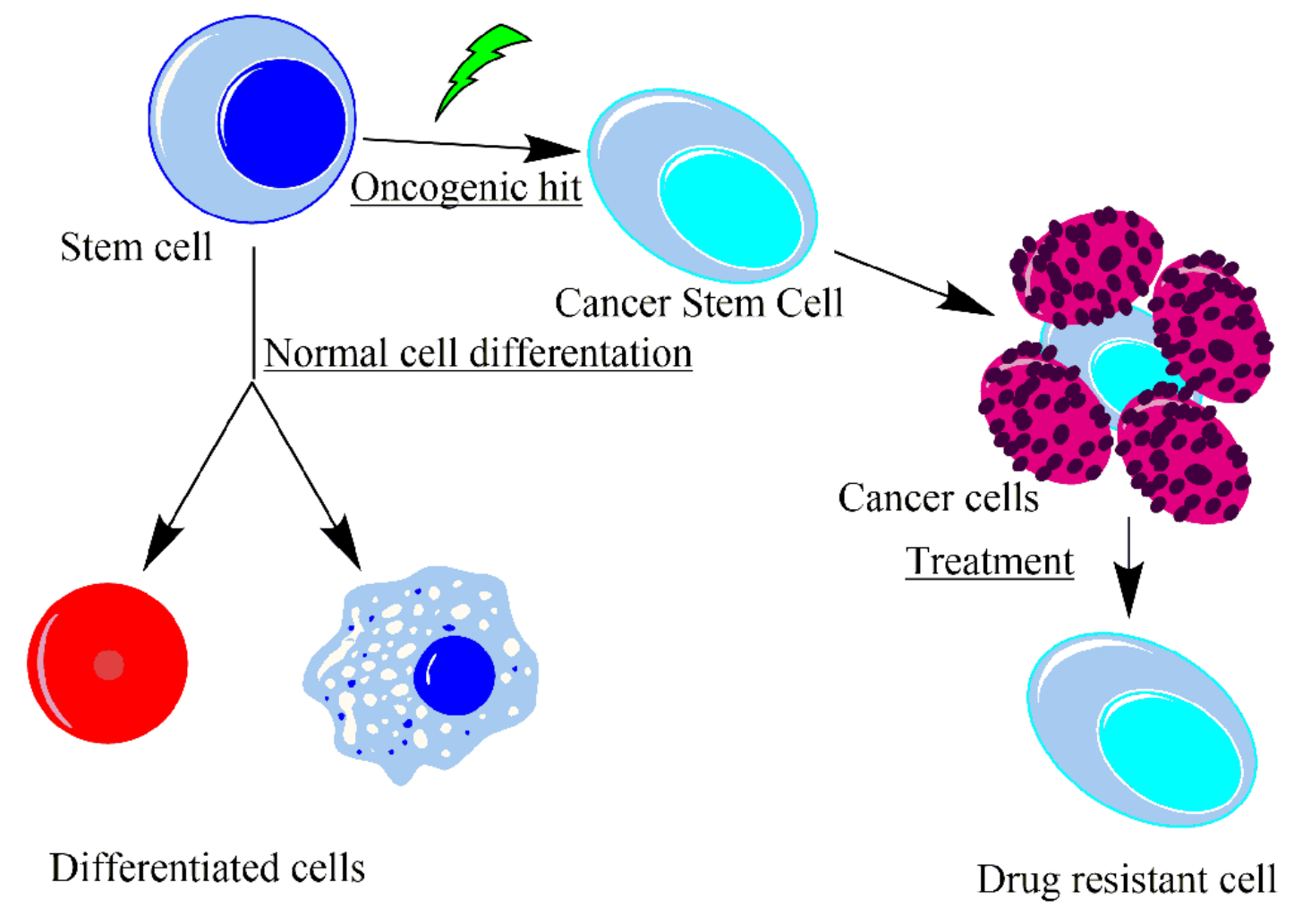

558 Figure 3. Cancer stem cells (CSCs) are derived during normal cell division process due to oncogenic hits 559 on the way. Stem cells undergo cell differentiation process for production of normal cells. Sometimes 560 these stem cells get an oncogenic hit and they stay as CSCs. Chemotherapy acts on all the other cancer 561 cells however, the CSCs are resistant to chemotherapy and hence retain their property to proliferate and 562 develop into cancer again. 